\title{
Nonlinear Frequency Modulated Signal Design
}

\author{
C. LEŚNIK* \\ Military University of Technology, Gen. S. Kaliskiego 2, 00-908 Warszawa, Poland
}

\begin{abstract}
The signal time-frequency structure designing is the important problem of radar system solution optimisation. The radar ambiguity function is the very useful signal characteristic that enables to know the signal potential possibilities. The effective numerical methods of the radar ambiguity function calculation give possibility for synthesis of the radar signal having expected features. The synthesis method concerning the nonlinear frequency modulation radar signal is presented in the paper. For the radar ambiguity function calculation the Zak transform is used in the method. The nonlinear frequency modulation signal model was described in which application limitations were taken into account. Examples of simulation research results are presented as a comment of the considerations.
\end{abstract}

PACS numbers: 84.40.Ua, 84.40.Xb

\section{Introduction}

The matched filter is the solution of the optimum radar signal processing problem. Optimum processing means maximizing the output signal-to-noise ratio (SNR) value. Such the filter is the very important part of the modern radar receiver. The filter output signal shape versus time is proportional to the transmit signal autocorrelation function $(\mathrm{ACF})$ in a case of full matching between signal and filter. The ACF shape determines the potential radar resolution in range and in radial velocity. Moreover, it describes the potential radar resistance for jamming. Non-zero radial velocity component of detected target causes mismatch being a result of the received signal Doppler frequency shift. As a result the output signal is deteriorated. The radar ambiguity function (RAF) is the very good tool, thanks to it the radar signal usefulness may be estimated. Effective numerical methods of the RAF calculation are the basis of the effective methods of the radar transmit signal synthesis $[1,2]$.

No acceptable high level of the time side lobes of the matched filter output signal having linear frequency modulation at input is the main feature of this very popular radar transmit signal. The side lobes presence makes detection of weak echo signals accompanied by strong signals from target with high radar cross-section (RCS) difficult or even impossible. The time side lobes suppression is realized thanks to weighting operation being a result of intended introduced mismatch to the filter. Unfortunately, such the operation causes moreover losses concerning the output signal that causes the radar range limitation among others. The nonlinear frequency modulation (NLFM) signal application is the other method for the time side lobe suppression. Such the method is lossless but for the signal synthesis it needs complicated

* e-mail: Czeslaw.Lesnik@wel.wat.edu.pl analytical tools. Moreover, the NLFM signal is more sensitive for the Doppler effect.

The NLFM signal synthesis method in which limitations relating the signal spectrum width are taken into consideration is described in the paper. In the method the Zak transform application for the RAF calculation and the stationary phase method used for a definite integral approximate solution are applied. The problem formulation and general description of the NLFM signal synthesis method based on the Zak transform are presented in Sect. 2. The precise analytic signal formula after modification connected with application limitations concerning the signal spectrum width is described in Sect. 3. Examples of simulation research results of the synthesized signal are presented in Sect. 4. Short conclusions are written in Sect. 5.

\section{NLFM signal design}

The RAF describes the complex output signal envelope versus delay time (it means in range) and in Doppler frequency shift (it means in radial velocity). In a case of the transmit signal $f(t)$ formula based on the signal parameters at the matched filter input and output is as follows:

$$
\chi\left(\tau, f_{\mathrm{d}}\right)=\int_{-\infty}^{\infty} f(t) f^{*}(t+\tau) \exp \left(-\mathrm{j} 2 \pi f_{\mathrm{d}} t\right) \mathrm{d} t,
$$

where $\tau$ and $f_{\mathrm{d}}$ are the output signal delay time and the input signal Doppler shift, respectively.

The Zak transform used in these considerations is defined as follows [3]. Let us assume that the function $f \in L^{2}(\boldsymbol{R})$ is known. The Zak transform $Z_{f}\{f(t)\}$ of the function $f(t)$ is called such the representation that $Z_{f}: f \rightarrow Z_{f}\{f\} \in L_{1}^{2}\left(\boldsymbol{R}^{2}\right)$ and $\forall x, y \in\langle 0,1\rangle$ is truth

$$
Z_{f}(x, y)=\sum_{k=-\infty}^{\infty} f(x+k) \exp (-\mathrm{j} 2 \pi k y) .
$$


One can show that between the signal RAF and its Zak transform there is relation described by the formula as follows [3]:

$$
\begin{gathered}
\chi_{f}\left(\tau, f_{\mathrm{d}}\right)=\int_{0}^{1} \int_{0}^{1}\left|Z_{f}(x, y)\right|^{2} \mathrm{e}^{-\mathrm{j} 2 \pi\left(f_{\mathrm{d}} x+\tau y\right)} \mathrm{d} x \mathrm{~d} y, \\
\tau, f_{\mathrm{d}} \in \boldsymbol{Z} .
\end{gathered}
$$

Based on (3) and assuming the integrand [3] periodic feature one can conclude that the RAF values in the integer points $\left(\tau, f_{\mathrm{d}}\right)$ represent the periodic function $\left|Z_{f}(x, y)\right|^{2}$ expansion coefficients into two-dimensional exponential Fourier series.

The start point of the considerations is the model of the signal being a sub-pulses train. The sub-pulses have the unitary duration time and are manipulated in amplitude and frequency. As a result one can obtain the function as follows:

$$
f(t)=\sum_{m=-M}^{M} \sum_{n=-N}^{N} a_{m n} \operatorname{rect}(t-m) \exp (\mathrm{j} 2 \pi n t),
$$

where $a_{m n}-$ elements of the matrix $A=$ $\left[a_{m n}\right]_{(2 M+1) \times(2 N+1)}$ that represents sub-pulses series coefficients, $m$ - the sub-pulse number, $n$ - the number of the frequency component in the $m$-th sub-pulse having the unitary minimum change in frequency from sub-pulse to sub-pulse and

$$
\operatorname{rect}[t-m]= \begin{cases}1 & \text { for }-0.5+m \leq t<0.5+m \\ 0 & \text { for elsewhere } t\end{cases}
$$

The synthesized NLFM signal $s(t)$ realizable in practice is the waveform with finite duration time, constant amplitude and continuous inner pulse frequency modulation. Its shape describes the formula as follows:

$$
s(t)=A \text { rect }\left[\frac{t-t_{i} / 2}{t_{i}}\right] \exp (\mathrm{j} 2 \pi \theta(t)),
$$

where $A$ - signal amplitude, $t_{i}$ - signal duration time and $\theta(t)$ - the angle modulation function looked for.

The signal synthesis problem means to find solution describing relationship between instantaneous frequency and time. Based on the signal formula (4) one should find connection between parameters $m$ and $n$ that describes relationship between time and the instantaneous signal frequency. Realizing the limit change from the parameters $m$ and $n$ integer set to the values interval from the real number set one can change from the case of frequency manipulation to the case of continuous frequency modulation.

Using periodic function based on the $k$-th Chebyshev polynomial one can prove that the Zak transform of the signal (4) is described by the formula as follows [3]:

$$
Z_{f}(x, y)=\cos 2 \pi k \sqrt{x^{2}+y^{2}} .
$$

The formula (5) can be generalized to the analytic class functions

$$
Z_{f}(x, y)=\exp \left(\mathrm{j} 2 \pi k\|(x, y)\|_{p}\right), \quad p>1,
$$

where $p-\operatorname{rank}$ of norm, $\|(x, y)\|_{p}=\sqrt[p]{x^{p}+y^{p}}$.
The Zak transform (6) periodicity gives opportunity to expansion it into two-dimensional exponential Fourier series

$$
Z_{f}(x, y)=\sum_{m=-\infty}^{\infty} \sum_{n=-\infty}^{\infty} a_{m n} \exp (\mathrm{j} \pi k(n x+m y))
$$

having expansion coefficients

$$
\begin{aligned}
& a_{m n} \\
& =\int_{0}^{1} \int_{0}^{1} \exp \left(-\mathrm{j} 2 \pi\left(n x+m y-k \sqrt[p]{x^{p}+y^{p}}\right)\right) \mathrm{d} x \mathrm{~d} y .
\end{aligned}
$$

The stationary phase principle is applied to describe the conditions that must be fulfilled by parameters $m$ and $n$ in order to reach the integrand function extreme values in the formula as above. In this way the connection between time and frequency is described thanks to the signal components have got dominant values. As a result the final formula of the angle modulation function [3] can be described

$$
\theta(t)=\left(k^{p^{\prime}}-t^{p^{\prime}}\right)^{\frac{1}{p^{\prime}}}, \quad t \in\langle-k, k\rangle,
$$

where

$$
p^{\prime}=\frac{p}{p-1} .
$$

After transformation done in order to achieve growing instantaneous frequency and the causal signal the wanted angle modulation function is described by

$$
\theta(t)=k-\left[k^{p^{\prime}}-\left(t-\frac{t_{i}}{2}\right)^{p^{\prime}}\right]^{\frac{1}{p^{\prime}}}, \quad t \in\langle 0,2 k\rangle .
$$

The final formula describing the causal NLFM signal formula is as follows:

$$
\begin{aligned}
& s(t)=A \operatorname{rect}\left(\frac{t-k}{2 k}\right) \\
& \times \exp \left(\mathrm{j} 2 \pi\left(k-\left(k^{p^{\prime}}-\left(t-\frac{t_{i}}{2}\right)^{p^{\prime}}\right)^{\frac{1}{p^{\prime}}}\right)\right), \\
& t \in\langle 0,2 k\rangle .
\end{aligned}
$$

The RAF function forming leads to the iterative procedure. As a result the parameters that fulfil their assumed features are precised.

\section{Modified NLFM signal}

The formula describing the synthesised signal instantaneous frequency is derived based on the derivative of Eq. (7). For example, for the $p=2$ one can achieve

$$
\theta(t)=k-\left[k^{2}-(t-k)^{2}\right]^{\frac{1}{2}}, \quad t \in\langle 0,2 k\rangle
$$

and

$$
f(t)=\frac{\mathrm{d} \theta(t)}{\mathrm{d} t}=\frac{t-k}{\sqrt{k^{2}-(t-k)^{2}}}, \quad t \in\langle 0,2 k\rangle .
$$

The infinite value of the frequency deviation is the important feature of the NLFM signal having instantaneous 
frequency described by the formula (10). It results from the infinity value of the $f(t)$ function at its domain both ends. That is why generation of such the signal is impossible.

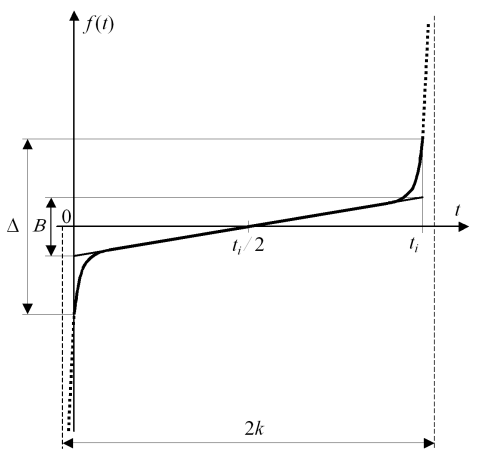

Fig. 1. Instantaneous frequency of the NLFM signal.

The theoretic pulse duration time $t_{i}=2 k$ was limited up to $t_{i}<2 k$ and the shift in time has changed its value from $k$ up to $t_{i} / 2$ both in order to eliminate the effect mentioned above. It is shown in Fig. 1. After taking into account modifications mentioned above the expressions (9) and (10) are as follows:

$$
\begin{aligned}
\theta(t) & =\frac{t_{i} \sqrt{\Delta^{2}+4}}{2 \Delta}-\left[\frac{t_{i}^{2}\left(\Delta^{2}+4\right)}{4 \Delta^{2}}-\left(t-\frac{t_{i}}{2}\right)^{2}\right]^{\frac{1}{2}}, \\
t & \in\left\langle 0, t_{i}\right\rangle, \\
f(t) & =\frac{t-\frac{t_{i}}{2}}{\sqrt{\frac{t_{i}^{2}\left(\Delta^{2}+4\right)}{4 \Delta^{2}}-\left(t-\frac{t_{i}}{2}\right)^{2}}}, \quad t \in\left\langle 0, t_{i}\right\rangle .
\end{aligned}
$$

That is why the NLFM signal model under condition of chosen parameters: $A, t_{i}$ and $\Delta$ is finally described by the formula as follows:

$$
\begin{aligned}
s(t) & =\operatorname{Arect}\left(\frac{t-t_{i} / 2}{t_{i}}\right) \exp \left(\mathrm { j } 2 \pi \left(\frac{t_{i} \sqrt{\Delta^{2}+4}}{2 \Delta}\right.\right. \\
& \left.\left.-\left(\frac{t_{i}^{2}\left(\Delta^{2}+4\right)}{4 \Delta^{2}}-\left(t-\frac{t_{i}}{2}\right)^{2}\right)^{\frac{1}{2}}\right)\right) .
\end{aligned}
$$

The instantaneous frequency change interval $\Delta$ determines the autocorrelation function of the NLFM signal side lobes level. The frequency change interval $B$ described by the linear part of the $f(t)$ characteristic (Fig. 1) determines first of all the main lobe width. This parameter value can be found as a result of the tangent to $f(t)$ equation description in the point $t_{i} / 2$. After calculations one can receive

$$
B=\frac{2 \Delta \sqrt{\Delta^{2}+4}}{\Delta^{2}+4} .
$$

For example the maximum, limiting parameter $B$ value is 2 , when the rank of norm is $p=2$.

The formulae from (11) to (14) are the basis of the simulation research of the synthesized signal features presented in the next part of the paper.

\section{Simulation results}

The theoretic research results are the basis for the NLFM signal generator simulation model design. It gives possibility to determine the basic signal characteristics and to test its features. Chosen simulation test results are shown on the sequence of figures.

The phase characteristic in radians of the synthesised NLFM signal is shown in Fig. 2. The signal was achieved under assumptions that: $\Delta=50 \mathrm{MHz}, t_{i}=50 \mu \mathrm{s}$. The time axis is normalized by the signal duration time $t_{i}$.

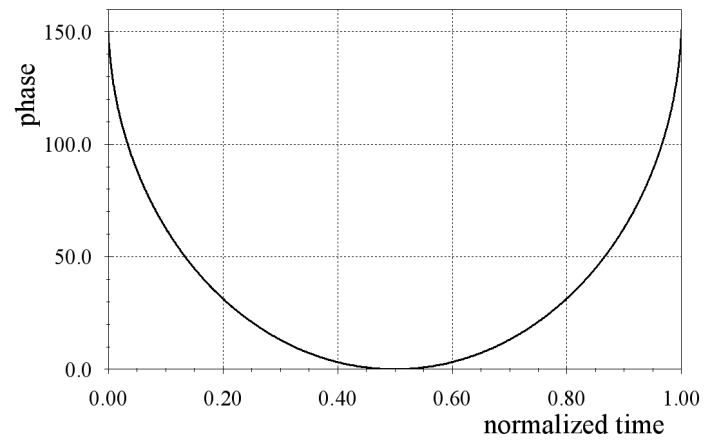

Fig. 2. Phase characteristic of the NLFM signal.

The frequency characteristic of the NLFM signal versus normalized time is presented in Fig. 3.

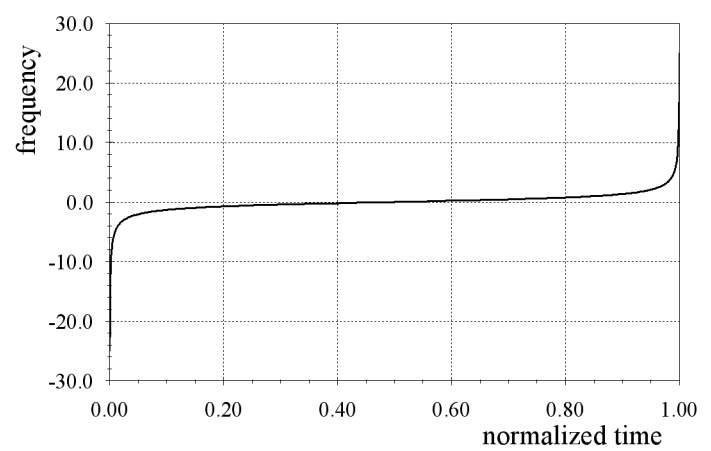

Fig. 3. Frequency characteristic of the NLFM signal.

Normalized spectrum magnitude of the NLFM signal versus frequency normalized by the sampling frequency is shown in Fig. 4. The spectrum magnitude of the LFM signal with the frequency deviation equal to the value of the $B$ parameter of the NLFM signal is presented also in Fig. 4 for comparison. The ACF of the both signals are shown in Fig. 5. The frequency axis is normalized by the sampling frequency.

It is easy to notice that the ACF side lobes level and their structure are much better in the case of the NLFM signal than in the case of LFM signal despite the main lobe broadening.

The most interesting are research results concerning the influence of the instantaneous frequency span $\Delta$ on 


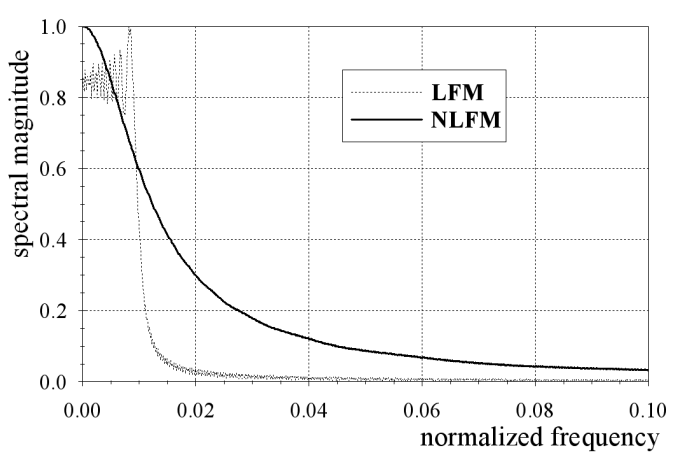

Fig. 4. Magnitude of the NLFM and LFM signals spectrum.

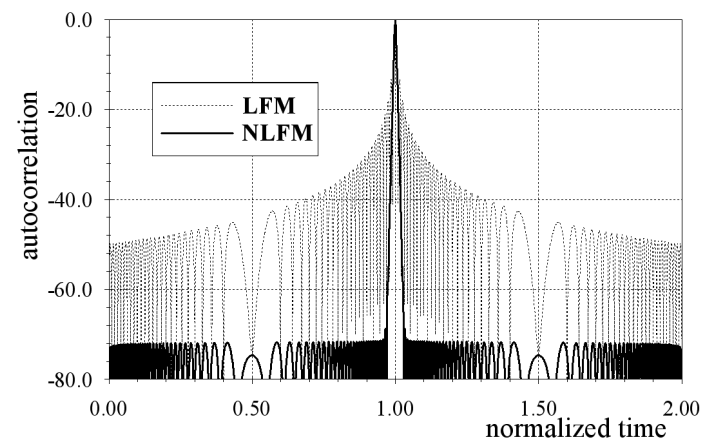

Fig. 5. ACF of the NLFM and LFM signals.

the main lobe width and side lobes level of the NLFM signal ACF. The research results are shown in Fig. 6 and Fig. 7. The frequency span axis is normalized by the frequency change interval $B$ described by the linear part of the $f(t)$ characteristic.

As a result of the parameter $\Delta$ changes the side lobes level control is possible in the relative wide interval. The cost of it is necessity of the radar receiver pass band broadening. The side lobes level suppression is achieved thanks to the main lobe duration broadening. It is measured at the maximum of the first side lobe level.

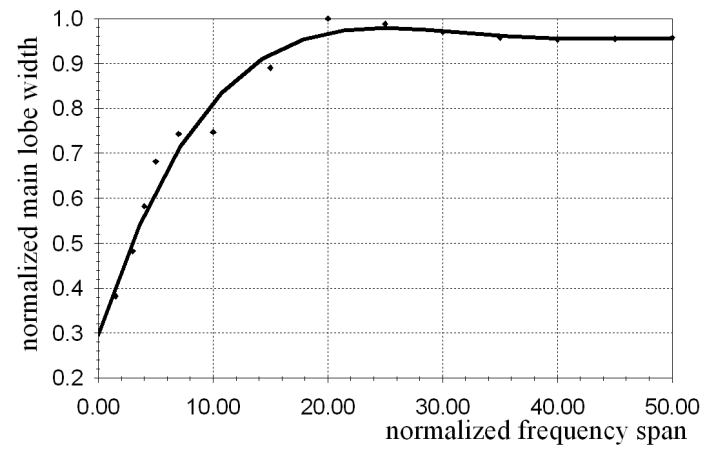

Fig. 6. Main lobe width of the NLFM signal ACF versus normalized frequency span.

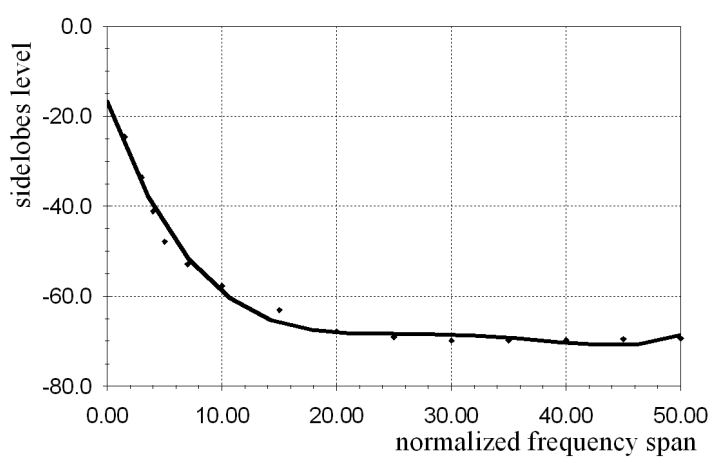

Fig. 7. Side lobes level of the NLFM signal ACF versus normalized frequency span.

\section{Summary}

Thanks to the method known from the literature [1-3] utilizing the NLFM signal synthesis method to describe the signal applicable model is possible. In the model the practical realization limits concerning the signal spectrum width are taken into account. Presented considerations are illustrated by the simulation research results. The possibility to control in the wide interval the signal side lobes level at the matched filter output is shown. It is achieved without any weighting loss operation. One should remember that the side lobes level is a result of compromise between its wanted value and the acceptable radar receiver pass band width.

\section{Acknowledgments}

This work was supported by the National Centre for Research and Development for the years 2007-2010 under Commissioned Research Project PBZ-MNiSWDBO-04/I/2007.

\section{References}

[1] Ch.E. Cook, M. Bernfeld, Radar Signals. An Introduction to Theory and Application, Artech House, Inc., Boston 1993.

[2] N. Levanon, E. Mozeson, Radar Signals, Wiley, Hoboken, New Jersey 2004.

[3] I. Gladkova, IEEE Trans. Aerospace Electron. Syst. 40, 1 (2004). 